\title{
Morphofunctional experience-dependent plasticity in the honeybee brain
}

\author{
Mara Andrione, ${ }^{1}$ Benjamin F. Timberlake, ${ }^{1}$ Giorgio Vallortigara, ${ }^{1}$ Renzo Antolini, ${ }^{1,2}$ \\ and Albrecht Haase ${ }^{1,2}$ \\ ${ }^{1}$ Center for Mind/Brain Sciences, University of Trento, 38068 Rovereto, Italy; ${ }^{2}$ Department of Physics, University of Trento, 38120 \\ Trento, Italy
}

\begin{abstract}
Repeated or prolonged exposure to an odorant without any positive or negative reinforcement produces experience-dependent plasticity, which results in habituation and latent inhibition. In the honeybee (Apis mellifera), it has been demonstrated that, even if the absolute neural representation of an odor in the primary olfactory center, the antennal lobe (AL), is not changed by repeated presentations, its relative representation with respect to unfamiliar stimuli is modified. In particular, the representation of a stimulus composed of a 50:50 mixture of a familiar and a novel odorant becomes more similar to that of the novel stimulus after repeated stimulus preexposure. In a calcium-imaging study, we found that the same functional effect develops following prolonged odor exposure. By analyzing the brains of the animals subjected to this procedure, we found that such functional changes are accompanied by morphological changes in the AL (i.e., a decrease in volume in specific glomeruli). The AL glomeruli that exhibited structural plasticity also modified their functional responses to the three stimuli (familiar odor, novel odor, binary mixture). We suggest a model in which rebalancing inhibition within the AL glomeruli may be sufficient to elicit structural and functional correlates of experience-dependent plasticity.
\end{abstract}

[Supplemental material is available for this article.]

The primary olfactory-processing center in the brain of the honeybee Apis mellifera, the antennal lobe (AL), is known to undergo functional plasticity changes in response to specific olfactory experiences. Classical appetitive conditioning has been extensively investigated for its potential to modify the representation of the rewarded odor in the AL (Sandoz 2011; Giurfa and Sandoz 2012). A number of calcium-imaging studies led to contradictory results (Faber et al. 1999; Sandoz et al. 2003; Peele et al. 2006; Hourcade et al. 2009; Denker et al. 2010) until it became clear that such changes are not absolute, but relative to the representation of nonrewarded odors (Faber et al. 1999; Rath et al. 2011). Thus, in accord with associative learning, the AL would act as a filter, increasing the relative salience of the rewarded odor pattern with respect to other stimuli. The resulting pattern would then be conveyed to higher brain areas, where the odor's valence is evaluated and behavioral responses are triggered (Chen et al. 2015). The reverse would happen in cases of nonassociative learning (Locatelli et al. 2013; Chen et al. 2015): an odor that is repeatedly presented to the bee olfactory system, associated with neither positive nor negative reinforcement, would lose relevance to the animal. The AL filter would reduce the relative salience of this odor response pattern with respect to others. In summary, following associative learning, previously rewarded components will have a competitive advantage in odor mixture representation over unrewarded ones, while, in the case of nonassociative learning, novel components will have an advantage over familiar ones (Locatelli et al. 2013; Chen et al. 2015). As a result, in nonassociative learning the preexposed stimulus becomes less likely to affect behavior, e.g., leading to overshadowing effects (Locatelli et al. 2013). In the case of prolonged rather than repeated exposure, in the fruit fly Drosophila melanogaster, a diminished olfactory avoidance of a previously habituated aversive odor-

\footnotetext{
Corresponding authors: albrecht.haase@unitn.it; mara.andrione@ unitn.it
}

Article is online at http://www.learnmem.org/cgi/doi/10.1101//m.046243.117. ant was observed (Manning 1967; Devaud et al. 2001; Sachse et al. 2007; Das et al. 2011). All these effects have been shown to be caused by an enhancement of inhibitory inputs from local neurons (LNs) to projection neurons (PNs) within the AL (Sachse et al. 2007; Das et al. 2011; Locatelli et al. 2013; Chen et al. 2015). In the case of associative learning, the relative increase in salience of the neural representation of the rewarded odor is due to octopamine-based modulation of LN activity (Sinakevitch et al. 2013; Chen et al. 2015). In the case of nonassociative experiences and habituation, the relative decrease in odor salience is dependent on recurrent inhibition on PNs via LNs (Sudhakaran et al. 2012). In the honeybee, both repeated (Chandra et al. 2010) and prolonged (Fernández et al. 2009) presentations of an unreinforced odor can produce latent inhibition, i.e., a delay in the acquisition of an associative memory of the same odor (Fernández et al. 2012). Latent inhibition has been suggested to rely on serotonin transmission (Fernández et al. 2012). Therefore, this neurotransmitter might also be involved in the mechanisms of "habituation," "activitydependent plasticity," or "central adaptation" previously described (Devaud et al. 2001; Sachse et al. 2007; Das et al. 2011). However, the site of action of serotonin responsible for latent inhibition was not precisely localized within the bee brain (Fernández et al. 2012). In the fruit fly, functional plasticity related to odor exposure was shown to be accompanied by volumetric changes at defined locations within the AL (Sachse et al. 2007; Das et al. 2011). These changes are believed to arise from increased branching and greater synaptic contact between LNs and PNs within the glomeruli (Sachse et al. 2007; Das et al. 2011). On the contrary, decreased

\footnotetext{
(C) 2017 Andrione et al. This article is distributed exclusively by Cold Spring Harbor Laboratory Press for the first 12 months after the full-issue publication date (see http://learnmem.cshlp.org/site/misc/terms.xhtml). After 12 months, it is available under a Creative Commons License (Attribution-NonCommercial 4.0 International), as described at http://creativecommons.org/licenses/by-nc/ $4.0 /$.
} 
glomerular volumes, observed in one study following odor exposure, were ascribed to synaptic loss (Devaud et al. 2001). Olfactory receptor neurons (ORNs), whose axons also contribute to glomerular connectivity, were shown in both cases to be morphologically and functionally unchanged by the procedure (Devaud et al. 2001; Sachse et al. 2007). In the honeybee, volumetric changes of specific AL glomeruli have been described in relation to age and foraging experience (Winnington et al. 1996; Sigg et al. 1997; Brown et al. 2004), or following associative learning-either at an early age (Arenas et al. 2012) or in foragers (Hourcade et al. 2009). However, structural plasticity related to nonassociative experiences within the AL has not yet been reported. Here, we investigated the effects of prolonged odor exposure on AL glomerular volumes collected via two-photon microscopy (Haase et al. 2010) and then tried to correlate the observed anatomical changes with functional modifications, observed via calcium imaging.

\section{Results}

To induce experience-dependent plasticity, bees were divided into three groups and preexposed over $3 \mathrm{~d}$ to one of two floral odorous compounds, 1-hexanol (HEX) and 1-nonanol (NNL), or to mineral oil as a stimulus control (CTR; for details see Materials and Methods). At the end of the 72-h procedure, a subset of bees was injected in their PNs with the calcium sensor fura- 2 and then kept overnight for another $18 \mathrm{~h}$ in the preexposure tents. The day after, stimulus-evoked activity was evaluated in a calcium-imaging experiment, in which each animal was presented with the single odors HEX and NNL and a binary 50:50 mixture (MIX). The change in fluorescence over time was analyzed for several glomeruli in $n=$ 5 bees of the HEX preexposed group, $n=4$ bees of the NNL preexposed group, and $n=5$ control bees. A subset of glomeruli was unambiguously identified within each bee. The activity patterns were averaged for single glomeruli over all individuals in which they could be identified within the three preexposure groups. These average glomerular response patterns show clear deviations among groups (Fig. 1). Recordings were limited to a single focal plane in each bee, to avoid odor overexposure by excessive repetition of the same paradigm of stimulation. Due to deviations among these focal planes in different animals, glomerular subsets were not fully overlapping across different bees. Moreover, some glomeruli within each recording could not be identified with full certainty.
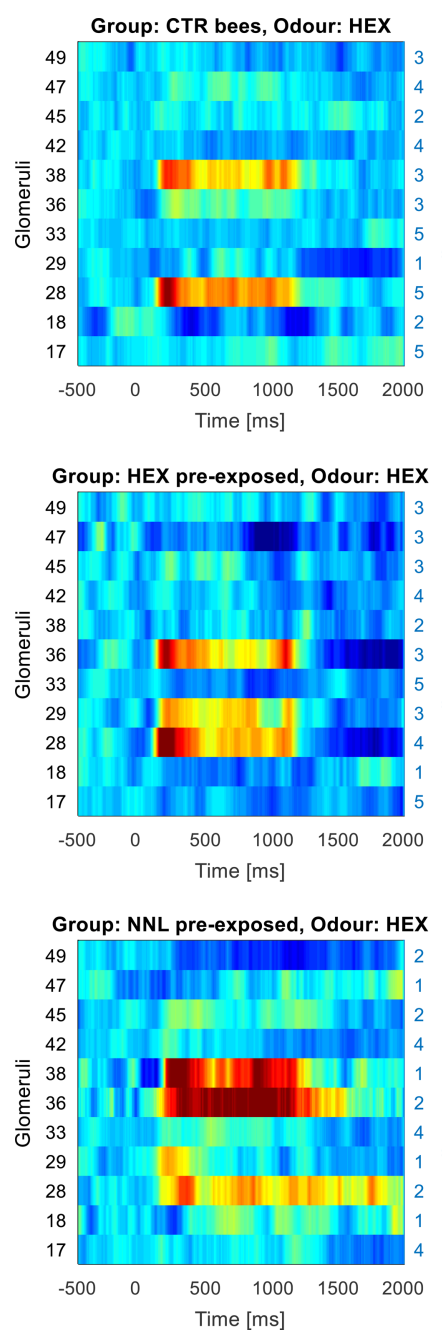
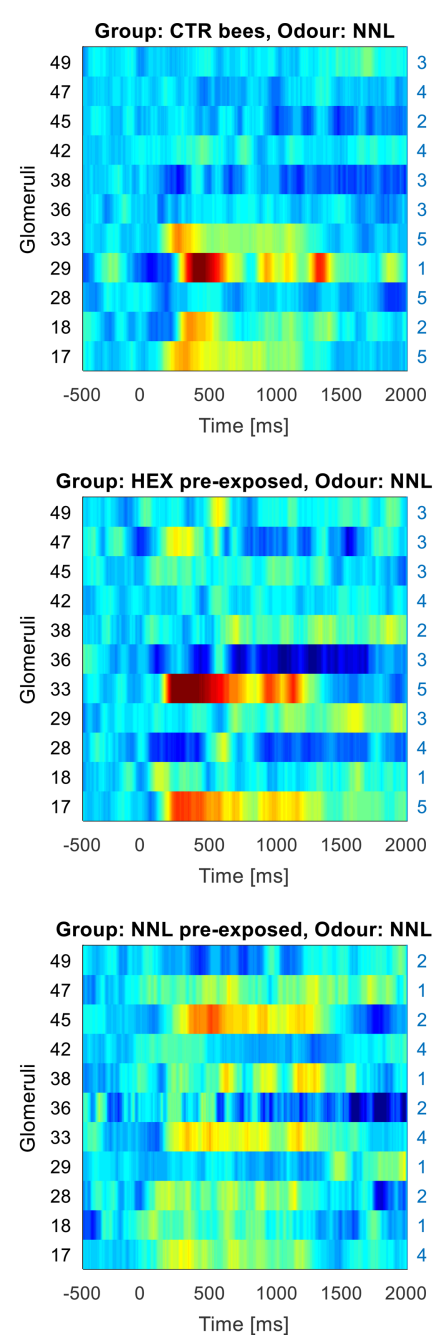
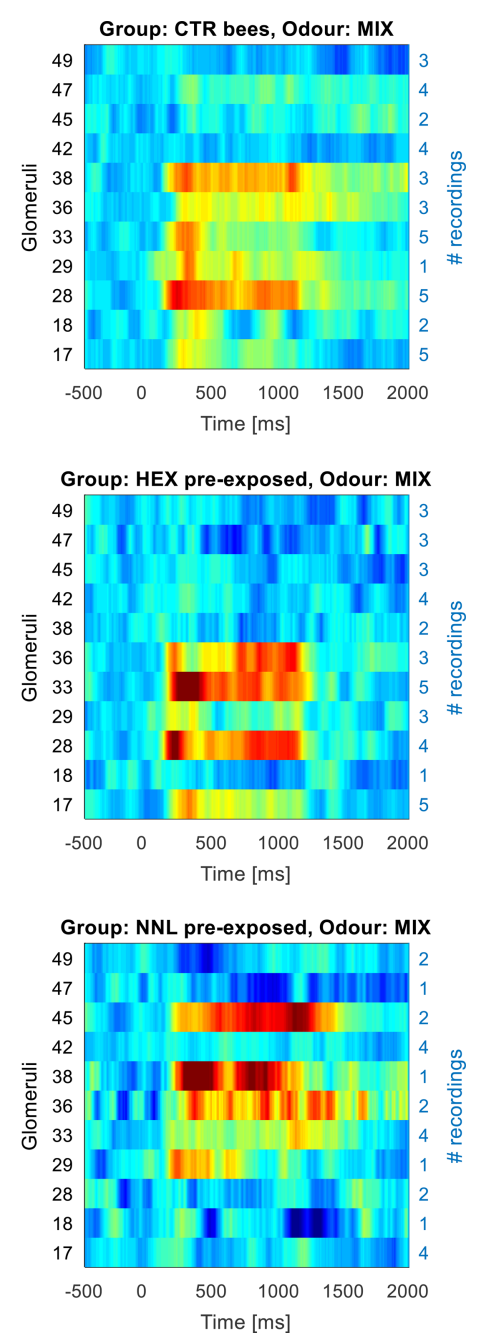

Figure 1. Fluorescence response maps of single glomeruli that were identified in at least one subject per group, averaged over subjects. Shown are background subtracted and normalized signals as a function of time from stimulus onset ( $-\Delta F / F$ ). The colormap reaches from inhibitive -0.1 (blue) to activated +0.2 (red). Figures within rows are responses to different odors within the same preexposed group. Columns show responses to the same odors in differently preexposed groups. The right $y$-axis shows in blue the number of subjects in which the glomerulus was identified and which therefore contributed to the averaging. 
Therefore, recorded data contained more information than those shown in Figure 1. To exploit the full glomerular response profile in each animal for further analysis, instead of averaging identified glomeruli only, glomeruli of all bees of the same treatment were pooled together to construct overall odor representations in each group $\left(n_{\mathrm{CTR}}=56, n_{\mathrm{HEX}}=62, n_{\mathrm{NNL}}=44\right)$. The dimensionality of this coding space was then reduced by principal component analysis (PCA). The odor-response dynamics (1 sec during stimulus and $1 \mathrm{sec}$ post-stimulus) are shown in the first two principal components (Fig. 2). The PCA shows a relative difference in odor representation among the three preexposed groups. In the CTR group, the binary mixture MIX activation vector is centered between the two pure components HEX and NNL, while in the odor preexposed groups, MIX shifts away from the familiar compound and toward the novel odor. This effect can be quantified by the Euclidean distance (ED) between odor pairs in the individual groups. The EDs were calculated based on the average activity of the recorded glomeruli in the interval 200 to $400 \mathrm{msec}$ after stimulus onset. We normalized the EDs within each group to the ED between the pure compounds (Fig. 3A). In the CTR group, in which animals were exposed to mineral oil only, single odors are again shown to be equidistant from the 50:50 mixture, while in the odor preexposed groups, the ED between mixture and novel odor is reduced. However, this difference was significantly different from zero only in the case of NNL preexposed bees (z-test of sampling distribution of differences, $P=0.016$; Fig. $3 \mathrm{~A}$, right).

To compare this relative shift among groups, we computed ratios

$$
\left(\mathrm{ED}_{\mathrm{HEX} \_\mathrm{MIX}}-\mathrm{ED}_{\text {NON_MIX }}\right) /\left(\mathrm{ED}_{\mathrm{HEX} \_\mathrm{MIX}}+\mathrm{ED}_{\mathrm{NON} \_\mathrm{MIX}}\right)
$$

within each group (Fig. 3B). This ratio was found to be approximately zero in the case of CTR bees, while it increased for HEX preexposed bees and decreased for NNL preexposed bees. The difference between the two preexposed groups was significant (z-test of sampling distribution of differences, $P=0.041$; Fig. 3B).

Since odor exposure continued over several days, we asked whether the observed functional plasticity was accompanied by structural changes. Therefore, following a fluorescent immunolabeling procedure of the synaptic compartments, brain morphology was imaged and analyzed for a subset of animals from each treatment group, sacrificed at the end of the 72-h exposure period. The AL glomeruli were identified, segmented, and 3D-reconstructed. A subset of glomeruli was bilaterally measured in each individual. The chosen glomeruli were selected for responding strongly either to HEX (glomeruli T1-38 and T1-28), to NNL (glomeruli T1-17 and T1-33) or to neither in particular (glomeruli T1-47 and T1-42), (see response maps in Fig. 1). Measured glomerular volumes are reported in Figure 4. A mixed ANOVA was performed, with right/left side and glomerulus number as within-subject factors and treatment as between-subject factor. Treatment, glomerulus, and their interaction significantly affected volumes (treatment: $F_{(2,40)}=7.8, P=0.0014$; glomerulus: $F_{(5,200)}=450, P<$ $10^{-106}$ : treatment $\times$ glomerulus: $\left.F_{(10,200)}=3.0, P=0.0017\right)$. Brain side did not show any significant main effect or interaction with the other factors (side: $F_{(1,40)}=0.060, P=0.81$; treatment $\times$ side: $F_{(2,40)}=1.4, \quad P=0.26 ;$ glomerulus $\times$ side: $F_{(5,200)}=0.29, \quad P=0.92$; treatment $\times$ glomerulus $\times$ side: $\left.F_{(10,200)}=0.80, P=0.63\right)$, meaning that the volumetric changes we observed were not lateralized (Haase et al. 2011; Rigosi et al. 2011; Frasnelli et al. 2014). Accordingly, for further independent sample $t$-test comparisons, corresponding right and left glomeruli were averaged in each bee. Odor exposure led to a decrease in volume of specific glomeruli. In particular, glomeruli T1-33 and T1-17 were both reduced in cases of NNL preexposure. A nonsignificant trend
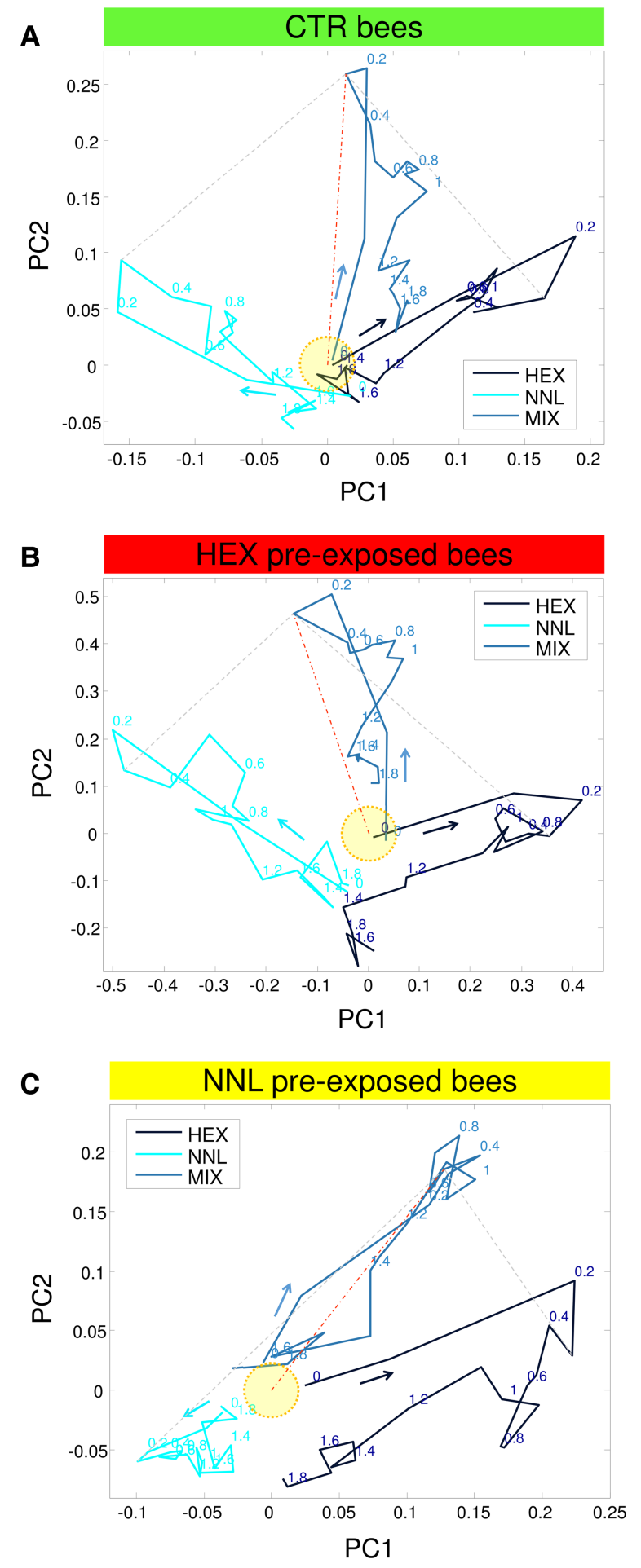

Figure 2. Activation dynamics during the presentation of three odor stimuli in the principal component coordinate system (1-sec stimulus and 1 -sec post-stimulus) for the three groups: $(A)$ control (CTR) bees, $(B)$ hexanol (HEX) preexposed bees, and (C) nonanol (NNL) preexposed bees. Activation vectors represent all measured glomeruli in a given treatment group. HEX activation vector is shown in blue, NNL in cyan, and their binary mixture (MIX) in light blue. The origin, marked by a yellow circle, corresponds to baseline activation. Arrows show the temporal axis; numbers indicate time after stimulus onset in seconds. Dashed gray lines connect the $300 \mathrm{msec}$ time-points, which are the points of maximal odor separation. A dotted and dashed orange line connects the origin with the binary mixture $300 \mathrm{msec}$ time-point. 


\section{A}

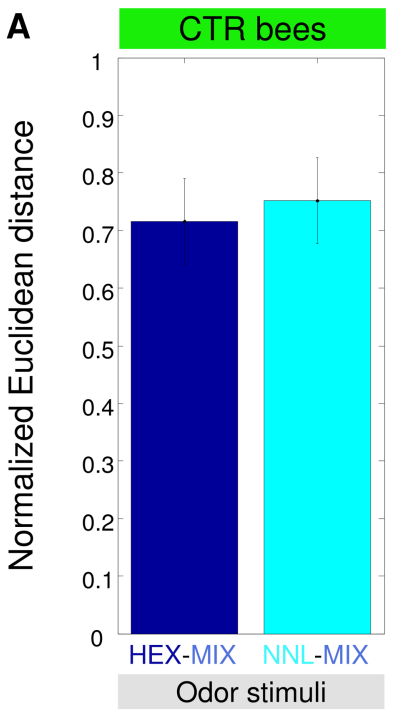

B

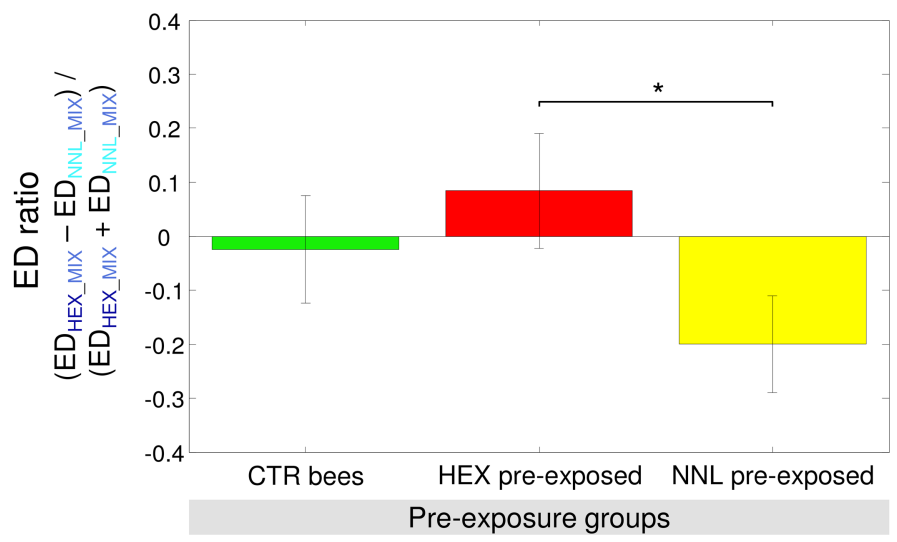

Figure 3. (A) Euclidean distances between representations of MIX and pure compounds within each group, normalized to the ED between the two pure compounds. Error bars represent standard deviations obtained via bootstrap resampling with replacement of glomeruli $(N=1000)$. The difference was significant in the NNL preexposed group ( $z$-test, $\left(^{*}\right) P=0.019$ ). (B) Ratios of normalized EDs $\left[\left(E D_{\text {HEX_MIX }}-E D_{\text {NON_MIX }}\right) /\left(E D_{\text {HEX_MIX }}+E D_{\text {NON_MIX }}\right)\right]$ within each group. Error bars represent standard deviations obtained by bootstrapping. The shift between the HEX and the NNL preexposed groups was significant ( $z$-test, $\left.\left({ }^{*}\right) P=0.041\right)$.

was also observed for a reduction in T1-28 volume in HEX preexposed bees.

Based on the observation that glomeruli T1-33 and T1-17 were most changed by odor preexposure, their single-odor-evoked response profiles were compared among groups (Fig. 5). T1-17 response profiles to the three stimuli HEX, NNL, and MIX are shown in Figure 5A; those of T1-33, in Figure 5B. Parts of the response pattern clearly changed following olfactory preexposure. In the CTR group (green bars), neither glomerulus responded to HEX (Fig. 5A,B left), but both were strongly excited by NNL (Fig. 5A,B center) and the MIX (Fig. 5A,B right). Glomeruli in the HEX preexposure group (red bars) developed a slightly inhibitory response to $\mathrm{HEX}$, although not significantly (Fig. 5A,B left). At the same time, the responses to NNL (Fig. 5A,B middle) and to the MIX (Fig. 5A,B right) increased in comparison to those of CTR bees (significantly in the case of glomerulus T1-33). Responses of the NNL preexposed group (yellow bars) showed slight changes in the opposite direction. The two preexposure groups gave rise to significantly different response to both NNL and MIX in the case of glomerulus T1-33 and to MIX in the case of glomerulus T1-17.

\section{Discussion}

We used a paradigm of prolonged preexposure of honeybees to single-odor compounds, similar to those that have been shown to produce measurable morphological and functional changes in D. melanogaster (Devaud et al. 2001; Sachse et al. 2007; Das et al. 2011). In the honeybee, repeated short stimulation with a single odorant induces a change in the relative representation of the binary mixture composed of this odor and a novel compound (Locatelli et al. 2013). Although our bees were treated instead with a continuous, long-term preexposure, functional imaging revealed the same effect, i.e., a suppression of the exposed odor representation in the odor response code of the mixture. This confirms the results of Locatelli et al. (2013) and shows that experience-dependent plasticity occurs in the honeybee not only during repeated exposure, but also during long-term exposure, as applied in our experiment. Interestingly, the latent inhibition of the proboscis extension response conditioning, a well-known consequence of unreinforced odor exposure in the honeybee, appears in cases of both repeated (Chandra et al. 2010) and prolonged (Fernández et al. 2009) odor exposure. On the other hand, we would exclude mechanisms of associative learning, as the paradigm of odor preexposure was specifically designed to avoid a spatial and completely synchronized temporal overlap between the two stimuli (odor, sucrose reward), i.e., besides being placed at opposite locations within the tents, the odor had no specific anticipatory value with respect to the sucrose. In fact, even if most of the time both stimuli were present, animals could experience periods with either only the odor or only the sucrose reward. Moreover, when the onset of the odor stimulus was preceding that of the sucrose reward, the interstimulus interval (ISI) was at least $1 \mathrm{~h}$, well past any suggested time scale for associative learning (Matsumoto et al. 2012).

Recently, Chakroborty et al. (2016) reported on changes in PN activity following $30 \mathrm{~min}$ adaptation of the olfactory system through exposure to complex mixtures. In our experiments, a long-duration preexposure was used to study the so-far-unaddressed issue of structural plasticity in relation to unrewarded odor exposure, which requires longer timescales.

The literature on prolonged odor exposure in Drosophila, suggests that odor-specific volumetric changes in the glomeruli would happen only during a certain time window following eclosion (Devaud et al. 2003). However, we observed structural plasticity in adult forager bees, well past any such window. The high flexibility that foraging activity requires, regarding individuation of food sources in space and time, may account for the preservation of plasticity in the honeybee olfactory structures.

Volumetric changes in the AL glomeruli have been reported in the adult bee alongside associative learning (Hourcade et al. 2009; Arenas et al. 2012). Here, we report, for the first time, 


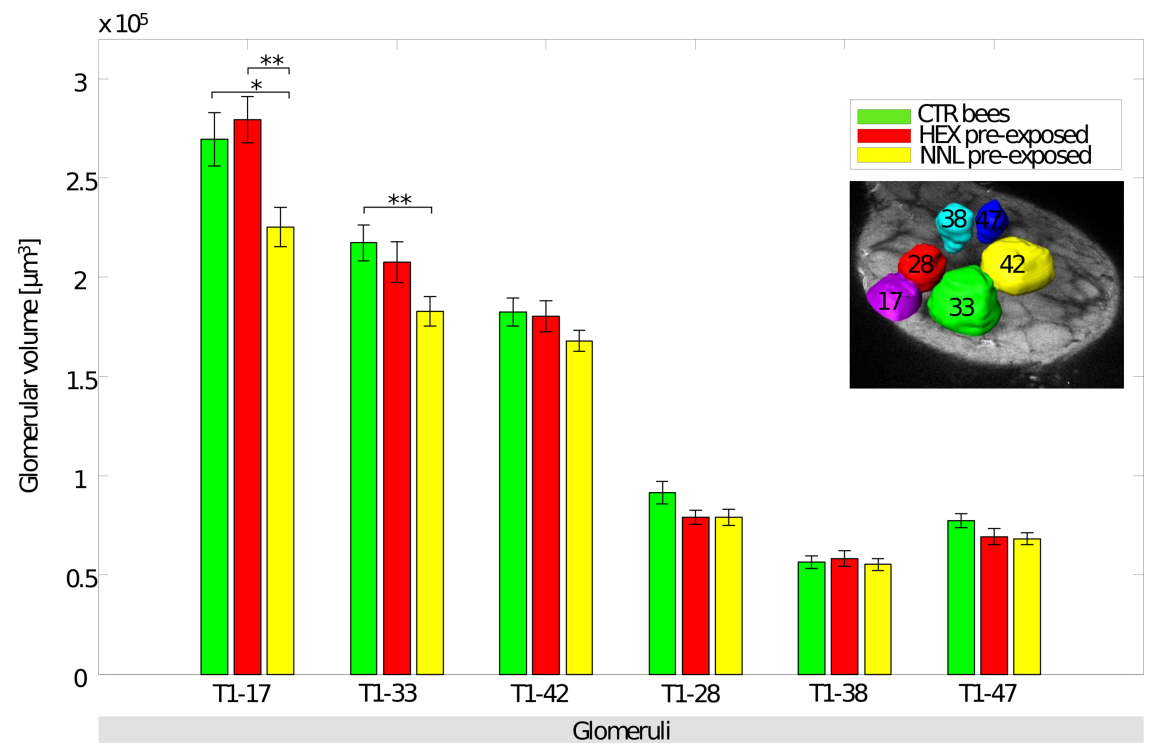

Figure 4. The average volumes of the six glomeruli in the three treatment groups (CTR bees, HEX preexposed, and NNL preexposed bees). An example of segmented and 3D-reconstructed glomeruli T1-17, $33,42,28,38,47$ from an immunolabeled left AL image stack is shown in the inset. Volume data were obtained by left-right averaging (see Materials and Methods). Error bars represent standard errors of the mean $\left(n_{\mathrm{CTR}}=13, n_{\mathrm{HEX}}=15, n_{\mathrm{NNL}}=15\right)$. Groups with significantly different means are indicated $\left(\left(^{*}\right) P<\right.$ $\left.0.05,\left({ }^{* *}\right) P<0.01\right)$.

volumetric changes accompanying unrewarded odor exposure. Our volumetric measurements on reconstructed glomeruli show significant changes in two of the six sampled glomeruli (T1-33 and T1-17) for one of the two preexposure odors (NNL). Adult activity-dependent plasticity, therefore, is able to produce similar volumetric changes irrespective of foraging experience before the experiment. It is likely that effects would be even stronger under experience- and age-controlled conditions.

The functional changes in those glomeruli showing structural plasticity (T1-17 and T1-33) suggest that the overall effects of unrewarded odor exposure on the odor code (Locatelli et al. 2013) are distributed across different glomeruli. In particular, we found significant differences in the response of glomeruli T1-17 and T1-33 to the binary mixture in the two preexposure groups. Moreover, trends can be observed, such as a slightly inhibitory HEX response for HEX preexposed bees in both T1-17 and T1-33, and a slight excitatory response to HEX in T1-33 for NNL preexposed bees. However, larger $n$ would be required to confirm these nonsignificant trends.

Functional effects observed in those two glomeruli, could be summarized the following way: the tuning of the T1-17 and T1-33 outputs (PNs), that normally signal NNL, became even sharper in the case of HEX preexposure and broader, that is, more accepting to odors other than NNL- in the case of NNL preexposure. Under this point of view, the case of glomerulus T1-17 is particularly interesting: in the CTR group, this glomerulus exhibited an excitatory response to NNL but no response to HEX, so one would expect the response to the binary mixture (MIX) to closely resemble the response to $\mathrm{NNL}$ in all groups. However, in the case of NNL preexposed bees, the presence of HEX seems to have dampened the response to NNL within the binary mixture, when compared with the response to NNL alone, causing an increase of mixture suppression (Deisig et al. 2006). Thus, the HEX component becomes more relevant to the MIX response of this glomerulus, even though in its pure form it did not elicit a response. Considering this, and the slight inhibitory response to HEX in the HEX preexposed group (both in T1-17 and T1-33), there seems to be a competition between NNL and HEX stimuli for the output activity of T1-17 and T1-33. This is not surprising, considering the high level of interconnectivity within the AL.

It is difficult to hypothesize which mechanism might have produced these shifts in odor response. We would exclude a major role of peripheral sensory adaptation, because the timescale needed for recovering such effect is usually minutes (Zufall and Leinders-Zufall 2000). The only animal in which longer-lasting adaptation was described $(3 \mathrm{~h})$ is the nematode C. elegans (Colbert and Bargmann 1995). At the time of imaging, our bees had not experienced the odor of preexposure since at least $1 \mathrm{~h}$. To our knowledge, such long adaptation recovery time has never been described in an insect.

A change in the connectivity of the $\mathrm{AL}$ seems to be the most probable reason for the functional plasticity observed. In particular, comparing functional data to volumetric changes, a modification at the level of ORNs or at the level of LNs can be hypothesized. A reduction of connections from ORNs to glomeruli T1-17 and T1-33 might cause a loss of tuning strength in the case of NNL preexposed bees and the reduction in volumes. Even if odor conditioning in the honeybee can modify olfactory receptor $(\mathrm{OR})$ expression in the periphery, nonassociative olfactory experience, such as pseudoconditioning, does not produce the same modifications (Claudianos et al. 2014). To our knowledge, data on prolonged odor exposure and its effect on ORN expression are missing in the honeybee. However, the hypothesis of a changing number or connectivity of ORN would be at odds with findings in Drosophila, where number, morphology, and physiology of ORNs were not affected by odor preexposure (Devaud et al. 2001; Sachse et al. 2007).

Alternatively, a decrease in volume at specific locations can be explained by a decrease in branching and synapses from inhibitory LNs onto PNs. This would be in accordance with results in Drosophila, where an enhancement of GABAergic transmission from LNs to active PNs (recurrent inhibition) followed odor preexposure (Sachse et al. 2007; Das et al. 2011; Sudhakaran et al. 2012). This enhancement, besides causing a volume increase, was necessary and sufficient to cause behavioral habituation (Das et al. 2011). In particular, one Drosophila study reported a volumetric increase following odor exposure in those glomeruli that are strongly activated by the preexposure odor, alongside with increased inhibition in the response of the same glomeruli to those odors (Sachse et al. 2007). Another study found a volume decrease of glomeruli without clear correlation to their involvement in the odor response (Devaud et al. 2001). We found the same effect in NNL preexposed bees in glomeruli T1-17 and T1-33, which are strongly activated by NNL (Fig. 1)

However, if a loss of inhibition underlies the volume loss in T1-17 and T1-33 in NNL-exposed bees, our functional results suggest that this favors the transmission of HEX, rather than NNL information. Responses to HEX and NNL in the honeybee are in fact far more distributed across glomeruli than the response to $\mathrm{CO}_{2}$ in the fruit fly AL (one responsive glomerulus; Sachse and Galizia 2002; Sachse et al. 2007). It might well be that standard ORN input (as in CTR bees) from a HEX stimulus reaches glomeruli T1-17 and T1-33 but is then strongly blocked, rather than passed on, to the 

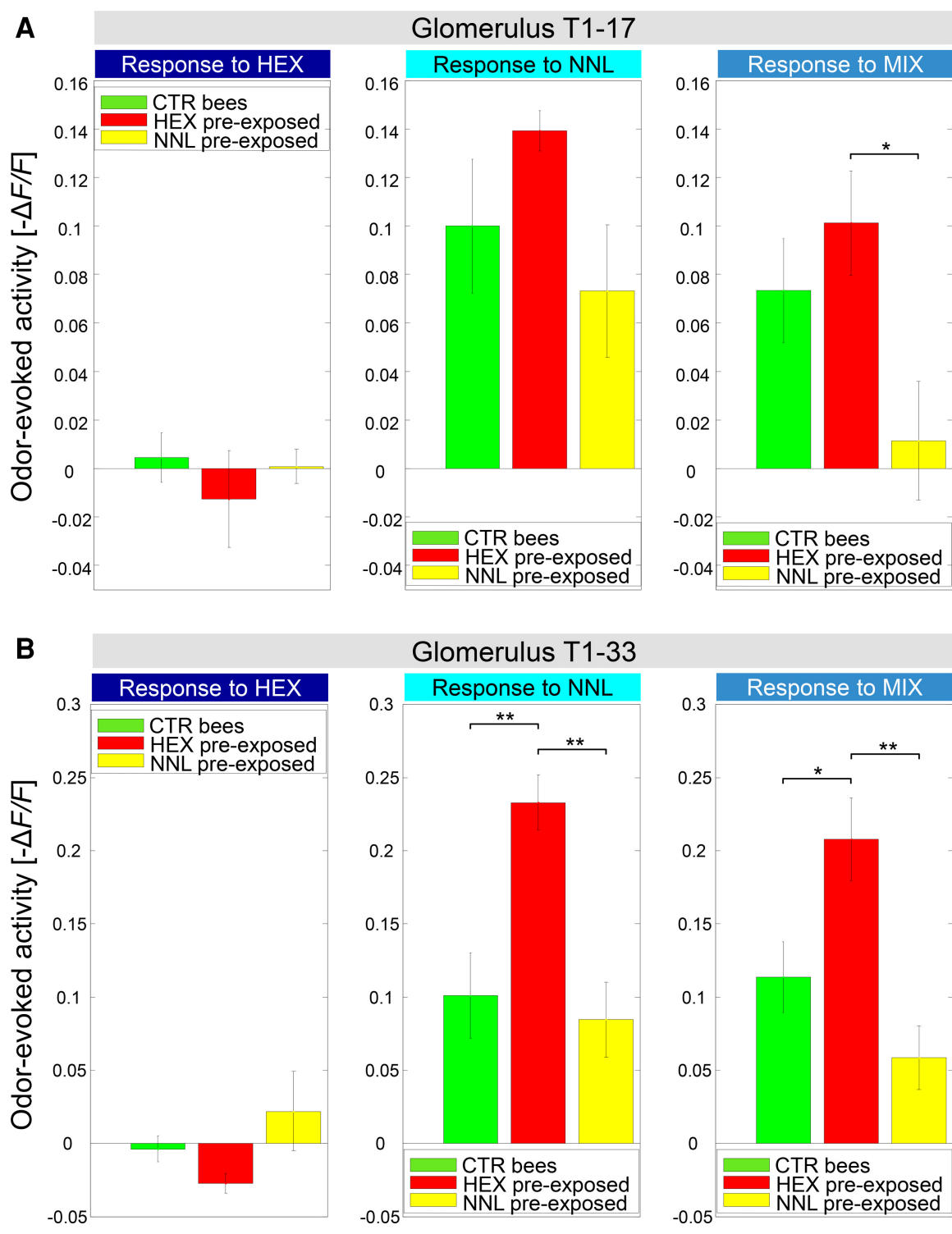

Figure 5. Odor-evoked activity of glomeruli T1-17 $(A)$ and T1-33 $(B)$ in the three treatment groups, averaged over single bees. Error bars represent standard errors of the mean $\left(n_{\mathrm{CTR}}=5, n_{\mathrm{HEX}}=5, n_{\mathrm{NNL}}=\right.$ 4). The left histograms show the responses to 1 -hexanol of all three treatment groups; the middle histograms, to 1-nonanol; and the right histograms, to the 50:50 mixture. The treatment effect was found to be significant via one-way ANOVA, and group means were confronted via two-sample $t$-tests $\left(\left(^{*}\right) P<\right.$ $\left.0.05,\left({ }^{* *}\right) P<0.01\right)$.

advantage of a contribution of NNL. This would be in accordance with a model in which functional inhibition, rather than stochastic or morphological, shapes the responses of PNs to similar odors (Linster et al. 2005). Inputs of ORNs activated by HEX and projecting to different glomeruli would indirectly prevent PNs of T1-33 and T1-17 from responding to the same odor, thus sharpening the response to NNL, as suggested elsewhere (Sachse and Galizia 2002). Similar phenomena are found in Drosophila (Silbering and Galizia 2007). This default blocking, or "occlusion," would be partially lost in our NNL preexposed bees, causing the MIX to be represented more similarly to HEX, and accompanied by volume loss in the glomeruli in question.

To conclude, we demonstrated that prolonged odor preexposure causes functional plasticity, shifting the relative representation of a binary mixture of the preexposure odor and a novel odor toward that novel compound. The odor of preexposure, losing functional relevance is partially suppressed in the mixture representation that is projected to higher brain centers. We identified single glomeruli causing these changes by measuring absolute differences in the responses to the two odors and their binary mixture, depending on the odor of preexposure. We observed for the first time accompanying structural plasticity in these glomeruli, manifested as a decrease in volume. We suggest as an underlying mechanism the loss of inhibition (or "occlusion") of the novel compound with respect to the preexposed odor.

\section{Materials and Methods}

\section{Animals}

Forager honeybees were collected with a transparent Plexiglas pyramid at the entrance of the hive in spring and summer 2015.

\section{Odor preexposure}

The bees were caged in groups of $\approx 30-40$ individuals in insect tents (BugDorm2120). The exposure procedure started at $4 \mathrm{pm}$ and lasted $72 \mathrm{~h}$. Odorants used were 1-hexanol (HEX) in one tent and 1-nonanol (NNL) in a second enclosure (all odors: 1:50 dilution in mineral oil up to a total volume of $100 \mu \mathrm{L}$ ). In a third tent, a control group was exposed to mineral oil only (all compounds from SigmaAldrich). All odor samples were suffused onto a piece of filter paper, enclosed in a plastic Petri dish with a perforated lid, and set inside each tent. Bees had access to a feeder in the form of a glass Boardman bottle containing 50\% sucrose solution seeping up through cylindrical dental filters. The feeder and the odor source were placed spatially separated in opposite corners of the tents (see Supplemental Material Fig. S1). An additional Petri dish carrying pure water was added at a third location to provide drinking water and to keep humidity at a constant level. The tents were kept in a single room at a distance of $\approx 3 \mathrm{~m}$ and were loosely covered with transparent plastic wrap in order to avoid excessive odor leakage. On the first day, bees were first provided with food and water. The odor dispenser was added after $1 \mathrm{~h}$. Each following day, food and odors were removed independently for certain periods, according to a daily schedule shown in Supplemental Figure S1, and renewed afterwards. The temperature and humidity of the room were monitored and kept, respectively, between $22^{\circ} \mathrm{C}$ and $26^{\circ} \mathrm{C}$ and $45 \%$ and $55 \%$. Lights were turned on in the morning and off in the evening (see Supplemental Material Fig. S1).

\section{Functional imaging protocol}

A subset of bees was prepared for in vivo calcium imaging. Briefly, after opening a cuticle window on top of the head, on one side of the brain, dextran-conjugated fura-2 (Thermo Fisher Scientific) was injected into the medial and lateral antenno-cerebral tracts $(\mathrm{m}$ - and l-ACTs) (Paoli et al. 2016b) via a pulled borosilicate glass needle. 
The next day, fluorescence changes in the ALs were recorded while stimulating the animals with 1-hexanol (HEX), 1-nonanol (NNL), and a binary 50:50 mixture of the two (MIX). Calcium-dependent fluorescence changes were acquired through repeated scanning (framerate $\approx 50 \mathrm{~Hz}$ ) of a $1 \mathrm{D}$ trace crossing all visible glomeruli ( $\approx 15-20)$ within one focal plane. This plane was chosen to contain the landmark glomeruli T1-17, T1-33, T1-42. For further details see Paoli et al. (2017).

\section{Odor stimuli}

During measurements, odor stimuli were delivered to the bee antennae through a constant air flow via a custom-made stimulus generator, controlled by a National Instruments board via a LabVIEW interface (Paoli et al. 2016a). The odor stimuli consisted in the head space of glass vials containing HEX diluted 1:500 or NNL diluted 1:250 (due to its lower volatility), both in mineral oil. The binary mixture was created by coherent summation of both individual channels. Each pure odor application had duration of $1 \mathrm{~s}$ and was repeated six times. The initial delay was $2 \mathrm{sec}$ for the novel odor and $8 \mathrm{sec}$ for preexposed odor. By using stimulus periods of $4 \mathrm{sec}$ for the novel odor and $2 \mathrm{sec}$ for the preexposed odor, a stimulus pattern is created which presents the novel odor, the preexposed odor, and the binary mixture in a pseudo-random manner (see Supplemental Material Fig. S2). CTR bees were randomly stimulated with the protocol of either HEX or NNL group.

\section{Exclusion of biases from different stimulation paradigms in different groups}

To exclude that the different order of odor presentation and the slightly different interstimulus intervals, rather than the preexposure procedure cause the different functional responses in the three groups, we compared odor-evoked responses in individual bees subjected to different stimulation paradigms (1 NNL preexposed bee, 1 HEX preexposed bee, and 2 CTR bees which received one or the other stimulation paradigm) in glomeruli T1-33 (Supplemental Fig. S3) and T1-17 (Supplemental Fig. S4). The responses to the MIX diverge between NNL preexposed and HEX preexposed animals, as we discuss in the Results section. Responses in CTR animals are similar to those of HEX preexposed bees (response to MIX = response to NNL), irrespective of the stimulation paradigms. This allows us to exclude major effects of the stimulation paradigm (order of presentation) on the recorded functional patterns.

\section{Functional data analysis}

Calcium-imaging data (fluorescence intensity along the scanline as a function of time) were post-processed using MATLAB (Mathworks). After denoising via spatial and temporal moving average filtering (Kernel size: $30 \mu \mathrm{m} \times 100 \mathrm{msec}$ ), responding regions were assigned to single glomeruli in a semi-automatic algorithm. The temporal signal was then split into single stimulus windows, from 0.5 -sec prestimulus to 1 -sec post-stimulus, for each glomerulus. The relative activation was obtained by normalizing the fluorescence intensity to the prestimulus baseline: $-\Delta F / F$. We averaged this signal over the three repetitions performed for every stimulus protocol in every subject. Only glomeruli responding with an average stimulus-evoked activity $(200-500 \mathrm{msec}$ after stimulus onset) significantly (1.96 $\sigma$ ) deviating from the average baseline activity were considered for quantitative analysis of the odor response pattern. The average $-\Delta F / F(200-400 \mathrm{msec}$ after stimulus onset) was used as a measure of maximum response. To analyze the separation of the odor response maps in glomerular coding space, the Euclidean distance ED between two odors $x$ and $y$ was calculated:

$$
\mathrm{ED}_{x, y}=\sqrt{\sum_{i=1}^{n}\left(x_{i}-y_{i}\right)^{2}}
$$

where $x_{i}$ and $y_{i}$ are the maximum responses of a single glomerulus $i$, which are summed over all $n$ glomeruli of all bees in all groups.
Normalized distances between pure odors and mixture were obtained by dividing their EDs by the ED between the pure compounds NNL and HEX within each group. The reliability of the EDs calculated on these large-dimension samples was assessed via bootstrap resampling with replacement ( $N=1000$ repetitions). The sampling distribution of differences between two EDs was then tested by a $z$-test against a standard normal distribution (after confirming its normality). A similar procedure was used to compare the ratios of the EDs. The ratio distributions were obtained by bootstrapping ( $N=1000$ repetitions), and each sampling distribution of differences between ratios was tested by a $z$-test against a standard normal distribution (after confirming its normality).

To visualize the response dynamics, the odor coding space dimension was reduced via a principal component analysis (PCA) of the joint coding space of all three groups. Original data dimension contain 486 variables (162 glomeruli $\times 3$ odors) and 180 samples (20 time points $\times 3$ trials $\times 3$ groups). Averaging over trials, groups, and the period 200-400 msec after stimulus onset provides the transformation matrix for the PCA. The first two principal components explain, respectively, $76.75 \%$ and $23.25 \%$ of the overall variation. The average odor dynamics within in each group was then quantified by bootstrapping ( $N=1000$ repetitions) subsets of $n=44$ glomeruli (the lowest $n$ of active glomeruli, recorded in NNL preexposed bees) belonging to each treatment group. Then, the weighted components relative to each group were averaged across $N$ and across all time points. The bootstrapping procedure was used to obtain balanced representations within the joint coding space even if number of original coding dimensions were different across groups. Single glomerular responses of T1-33 and T1-17 to the odor stimuli were compared among treatment groups via one-way ANOVA and independent sample $t$-tests. All preprocessing steps and analyses were conducted in MATLAB.

\section{Brain dissociation and immunohistochemistry}

After $72 \mathrm{~h}$ of odor exposure, a subset of bees was sacrificed, and brains were dissociated and processed for immunohistochemistry. Briefly, bees were decapitated and heads were fixed in a $4 \%$ paraformaldehyde solution $\left(4^{\circ} \mathrm{C} \mathrm{O} / \mathrm{N}\right)$. Brains were later carefully dissociated in PBS. Subsequent washing and immunostaining procedures were all conducted in a $0.5 \%$ Triton X-100 PBS solution. After blocking of nonspecific sites through incubation in a $5 \%$ normal goat serum solution ( $1 \mathrm{~h} \mathrm{RT})$, the brains were incubated $\left(48 \mathrm{~h}, 4^{\circ} \mathrm{C}, 4 \%\right.$ in Triton-PBS) with anti-synapsin antibodies (DSHB Hybridoma Product 3C11 (anti SYNORF1)). The binding sites were then revealed with an Alexa Fluor 546 secondary fluorescent antibody (Alexa Fluor 546 donkey anti-mouse IgG $(\mathrm{H}+\mathrm{L})$, Invitrogen Molecular Probes; incubation $48 \mathrm{~h}, 4^{\circ} \mathrm{C}, 2 \%$ in Triton-PBS).

\section{Optical imaging setup}

Both morphological and functional images were acquired via twophoton microscopy (Ultima IV, Prairie Technologies-Bruker), equipped with an ultra-short pulsed laser (Mai Tai Deep See HP, Spectra-Physics-Newport) as an excitation source. Excitation wavelength was $800 \mathrm{~nm}$, fluorescence was separated by a dichroic beam-splitter, filtered by a $70 \mathrm{~nm}$ bandpass filter centered at 525 nm (both Chroma), and detected by Photomultiplier tubes (Hamamatsu Photonics). Excitation and signal collection were performed in epifluorescence configuration via a water-immersion objective (20x, NA = 1, Olympus). Immersion liquid was Triton-PBS for ex vivo and Ringer's solution (Galizia and Vetter 2004) for in vivo imaging.

\section{Data analysis volumetric data}

$Z$-stacks of the right and left ALs ( $3 \mu \mathrm{m}$ step size along the anteroposterior axis) were evaluated in Image (Schneider et al. 2012), and glomeruli were identified. In cases where both the right and the left sides were perfectly intact and unambiguous labeling of the glomeruli could be achieved, the images were processed in Amira (FEI-Thermo Fisher Scientific) for 3D reconstruction and 
volumetric measurements of the glomeruli of interest. The whole procedure was conducted blindly with respect to both group and side, necessitating prior horizontal flipping of $z$-stacks of the right AL.

Volumes were analyzed via a mixed ANOVA with side and glomerulus as within-subject factors and treatment as betweensubject factor. Each glomerulus was then analyzed via repeatedmeasures ANOVA with side as within-subject factor and treatment as between-subject factor. Independent sample $t$-test comparisons were performed within each glomerulus, using a right-left averaged measure of each glomerulus in each bee. All analyses were conducted in MATLAB.

\section{Acknowledgments}

We acknowledge financial support by the Autonomous Province of Trento (Research unit IBRAIM) and the Autonomous Province of Bolzano (project B26J16000310003).

\section{References}

Arenas A, Giurfa M, Sandoz JC, Hourcade B, Devaud JM, Farina WM. 2012. Early olfactory experience induces structural changes in the primary olfactory center of an insect brain. Eur J Neurosci 35: 682-690.

Brown SM, Napper RM, Mercer AR. 2004. Foraging experience, glomerulus volume, and synapse number: a stereological study of the honey bee antennal lobe. J Neurobiol 60: 40-50.

Chakroborty NK, Menzel R, Schubert M. 2016. Environment specific modulation of odorant representations in the honeybee brain. Eur J Neurosci 44: 3080-3093.

Chandra SBC, Wright GA, Smith BH. 2010. Latent inhibition in the honey bee, Apis mellifera: is it a unitary phenomenon? Anim Cogn 13: 805-815.

Chen J-Y, Marachlian E, Assisi C, Huerta R, Smith BH, Locatelli F, Bazhenov M. 2015. Learning modifies odor mixture processing to improve detection of relevant components. J Neurosci 35: 179-197.

Claudianos C, Lim J, Young M, Yan S, Cristino AS, Newcomb RD, Gunasekaran N, Reinhard J. 2014. Odor memories regulate olfactory receptor expression in the sensory periphery. Eur J Neurosci 39: 1642-1654.

Colbert HA, Bargmann CI. 1995. Odorant-specific adaptation pathways generate olfactory plasticity in C. elegans. Neuron 14: 803-812.

Das S, Sadanandappa MK, Dervan A, Larkin A, Lee JA, Sudhakaran IP, Priya R, Heidari R, Holohan EE, Pimentel A, et al. 2011. Plasticity of local GABAergic interneurons drives olfactory habituation. Proc Natl Acad Sci 108: E646-E654.

Deisig N, Giurfa M, Lachnit H, Sandoz J-C. 2006. Neural representation of olfactory mixtures in the honeybee antennal lobe. Eur J Neurosci 24: 1161-1174.

Denker M, Finke R, Schaupp F, Grün S, Menzel R. 2010. Neural correlates of odor learning in the honeybee antennal lobe. Eur J Neurosci 31: 119-133.

Devaud JM, Acebes A, Ferrús A. 2001. Odor exposure causes central adaptation and morphological changes in selected olfactory glomeruli in Drosophila. J Neurosci 21: 6274-6282.

Devaud J-M, Acebes A, Ramaswami M, Ferrús A. 2003. Structural and functional changes in the olfactory pathway of adult Drosophila take place at a critical age. J Neurobiol 56: 13-23.

Faber T, Joerges J, Menzel R. 1999. Associative learning modifies neural representations of odors in the insect brain. Nat Neurosci 2: 74-78.

Fernández VM, Arenas A, Farina WM. 2009. Volatile exposure within the honeybee hive and its effect on olfactory discrimination. J Comp Physiol A Neuroethol Sens Neural Behav Physiol 195: 759-768.

Fernández VM, Giurfa M, Devaud J-M, Farina WM. 2012. Latent inhibition in an insect: the role of aminergic signaling. Learn Mem 19: 593-597.

Frasnelli E, Haase A, Rigosi E, Anfora G, Rogers LJ, Vallortigara G. 2014. The bee as a model to investigate brain and behavioural asymmetries. Insects 5: $120-138$.

Galizia GC, Vetter RS. 2004. Methods in insect sensory neuroscience. CRC press, Boca Raton.

Giurfa M, Sandoz J-C. 2012. Invertebrate learning and memory: fifty years of olfactory conditioning of the proboscis extension response in honeybees. Learn Mem 19: 54-66.
Haase A, Rigosi E, Trona F, Anfora G, Vallortigara G, Antolini R, Vinegoni C. 2010. In-vivo two-photon imaging of the honey bee antennal lobe. Biomed Opt Express 2: 131-138.

Haase A, Rigosi E, Frasnelli E, Trona F, Tessarolo F, Vinegoni C, Anfora G Vallortigara G, Antolini R. 2011. A multimodal approach for tracing lateralisation along the olfactory pathway in the honeybee through electrophysiological recordings, morpho-functional imaging, and behavioural studies. Eur Biophys J 40: 1247-1258.

Hourcade B, Perisse E, Devaud J-M, Sandoz J-C. 2009. Long-term memory shapes the primary olfactory center of an insect brain. Learn Mem 16: 607-615.

Linster C, Sachse S, Galizia CG. 2005. Computational modeling suggests that response properties rather than spatial position determine connectivity between olfactory glomeruli. INeurophysiol 93: 3410-3417.

Locatelli FF, Fernandez PC, Villareal F, Muezzinoglu K, Huerta R, Galizia CG, Smith BH. 2013. Nonassociative plasticity alters competitive interactions among mixture components in early olfactory processing. Eur J Neurosci 37: 63-79.

Manning A. 1967. "Pre-imaginal" conditioning in Drosophila. Nature 216: 338-340.

Matsumoto Y, Menzel R, Sandoz J-C, Giurfa M. 2012. Revisiting olfactory classical conditioning of the proboscis extension response in honey bees: a step towards standardized procedures. J Neurosci Methods 211: 159-167.

Paoli M, Anesi A, Antolini R, Guella G, Vallortigara G, Haase A. 2016a. Differential odour coding of isotopomers in the honeybee brain. Sci Rep 6: 21893.

Paoli M, Weisz N, Antolini R, Haase A. 2016b. Spatially resolved time-frequency analysis of odour coding in the insect antennal lobe. Eur J Neurosci 44: 2387-2395.

Paoli M, Andrione M, Haase A. 2017. Imaging Techniques in Insects. In Lateralized brain functions, 1st ed. (ed. Rogers LJ, Vallortigara G), pp. 471-519. Springer, New York, NY.

Peele P, Ditzen M, Menzel R, Galizia CG. 2006. Appetitive odor learning does not change olfactory coding in a subpopulation of honeybee antennal lobe neurons. J Comp Physiol A 192: 1083-1103.

Rath L, Galizia GC, Szyszka P. 2011. Multiple memory traces after associative learning in the honey bee antennal lobe. Eur J Neurosci 34: 352-360.

Rigosi E, Frasnelli E, Vinegoni C, Antolini R, Anfora G, Vallortigara G, Haase A. 2011. Searching for anatomical correlates of olfactory lateralization in the honeybee antennal lobes: a morphological and behavioural study. Behav Brain Res 221: 290-294.

Sachse S, Galizia CG. 2002. Role of inhibition for temporal and spatial odor representation in olfactory output neurons: a calcium imaging study. $J$ Neurophysiol 87: 1106-1117.

Sachse S, Rueckert E, Keller A, Okada R, Tanaka NK, Ito K, Vosshall LB. 2007. Activity-dependent plasticity in an olfactory circuit. Neuron 56: 838-850.

Sandoz JC. 2011. Behavioral and neurophysiological study of olfactory perception and learning in honeybees. Front Syst Neurosci 5: 98.

Sandoz J-C, Galizia CG, Menzel R. 2003. Side-specific olfactory conditioning leads to more specific odor representation between sides but not within sides in the honeybee antennal lobes. Neuroscience 120: 1137-1148.

Schneider CA, Rasband WS, Eliceiri KW. 2012. NIH Image to ImageJ: 25 years of image analysis. Nat Methods 9: 671-675.

Sigg D, Thompson CM, Mercer AR. 1997. Activity-dependent changes to the brain and behavior of the honey bee, Apis mellifera (L.). J Neurosci 17: $7148-7156$

Silbering AF, Galizia CG. 2007. Processing of odor mixtures in the Drosophila antennal lobe reveals both global inhibition and glomerulus-specific interactions. J Neurosci 27: 11966-11977.

Sinakevitch IT, Smith AN, Locatelli F, Huerta R, Bazhenov M, Smith BH. 2013. Apis mellifera octopamine receptor 1 (AmOA1) expression in antennal lobe networks of the honey bee (Apis mellifera) and fruit fly (Drosophila melanogaster). Front Syst Neurosci 7: 70.

Sudhakaran IP, Holohan EE, Osman S, Rodrigues V, Vijayraghavan K, Ramaswami M. 2012. Plasticity of recurrent inhibition in the Drosophila antennal lobe. J Neurosci 32: 7225-7231.

Winnington AP, Napper RM, Mercer AR. 1996. Structural plasticity of identified glomeruli in the antennal lobes of the adult worker honey bee. J Comp Neurol 365: 479-490.

Zufall F, Leinders-Zufall T. 2000. The cellular and molecular basis of odor adaptation. Chem Senses 25: 473-481.

Received June 29, 2017; accepted in revised form August 11, 2017. 


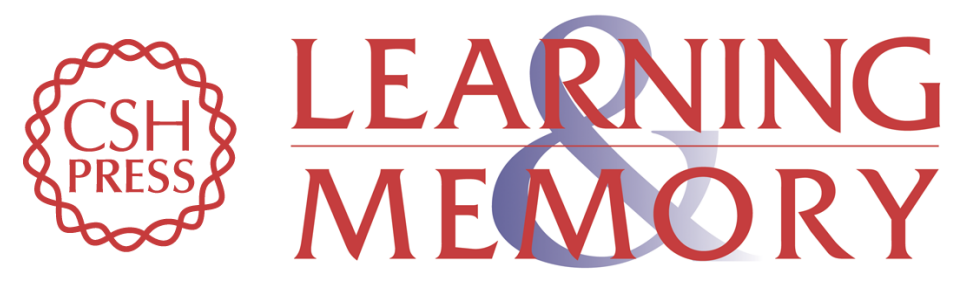

\section{Morphofunctional experience-dependent plasticity in the honeybee brain}

Mara Andrione, Benjamin F. Timberlake, Giorgio Vallortigara, et al.

Learn. Mem. 2017, 24:

Access the most recent version at doi:10.1101/Im.046243.117

\section{Supplemental http://learnmem.cshlp.org/content/suppl/2017/11/14/24.12.622.DC1 Material}

References This article cites 40 articles, 9 of which can be accessed free at: http://learnmem.cshlp.org/content/24/12/622.full.html\#ref-list-1

Creative This article is distributed exclusively by Cold Spring Harbor Laboratory Press for the Commons first 12 months after the full-issue publication date (see

License http://learnmem.cshlp.org/site/misc/terms.xhtml). After 12 months, it is available under a Creative Commons License (Attribution-NonCommercial 4.0 International), as described at http://creativecommons.org/licenses/by-nc/4.0/.

Email Alerting Receive free email alerts when new articles cite this article - sign up in the box at the Service top right corner of the article or click here. 